\title{
DESIGN, CONSTRUCTION AND STATUS OF ALL NIOBIUM SUPERCONDUCTING PHOTOINJECTOR AT BNL
}

\author{
T. Srinivasan-Rao, I. Ben-Zvi, A. Burrill, G. Citver, A. Hershcovitch, D. Pate, A. Reuter, J. Scaduto, \\ Q. Zhao, Y. Zhao, BNL, Upton, NY \\ J. Delayen, P. Kneisel, TJNAF, Newport News, VA
}

\begin{abstract}
H. Bluem, M. Cole, A. Favale, J. Rathke, T. Schultheiss, Advanced Energy Systems, Medford, NY
\section{Abstract}

We present here the design and construction of an all niobium superconducting RF injector to generate high average current, high brightness electron beam. A $1 / 2$ cell superconducting cavity has been designed, built, and tested. A cryostat has been built to cool the cavity to $\sim 2 \mathrm{~K}$. The RF system can deliver up to $500 \mathrm{~W}$ at $1.3 \mathrm{GHz}$ to the cavity. A mode-locked $\mathrm{Nd}: \mathrm{YVO}_{4}$ laser, operating at 266 $\mathrm{nm}$ with $0.15 \mathrm{~W}$ average power, phase locked to the RF,

The laser is reflected by an off axis metallic mirror into the beam line to irradiate the cathode. The electron beam generated by the laser is transported through a solenoid to the Faraday cup at the end of the beam line. The beam envelope simulated using PARMELA is shown in Figure 2. The energy and longitudinal and transverse emittances of a electron bunch with $1 \mathrm{pC}$ charge from $1 \mathrm{~mm}$ spot size and $6 \mathrm{ps}$ duration, measured at the exit of the gun is shown in Figure 3 for different launch phases
\end{abstract} will irradiate a laser cleaned $\mathrm{Nb}$ surface at the back wall of the cavity. Description of critical components and their status are presented in the paper. Based on DC measurements, QE of up to $10^{-4}$ can be expected from such cavity

\section{INTRODUCTION}

In recent years, there had been considerable interest in developing high average current, high brightness electron beams both for accelerator and photon source applications. With its low power requirements a superconducting RF (SCRF) photoinjector is an ideal electron source for these applications. However, the low quantum efficiency of $\mathrm{Nb}$ and the short lifetime and complicated design associated with alkali cathodes have slowed the implementation of this scheme. Recently, it has been shown that the $\mathrm{QE}$ of $\mathrm{Nb}$ can be improved significantly by laser cleaning it in vacuum ${ }^{[1]}$. A feasibility study to incorporate this technique in the surface preparation of SCRF injector and generate high average current electron beam was undertaken and the results are presented in the following sections.

\section{SIMULATIONS}

A schematic of the cavity, cryostat and electron beam transport are shown in Figure 1.

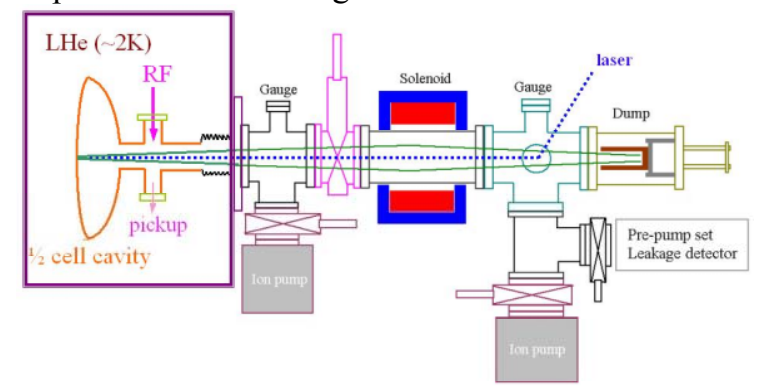

Figure 1: Schematic of the beam transport

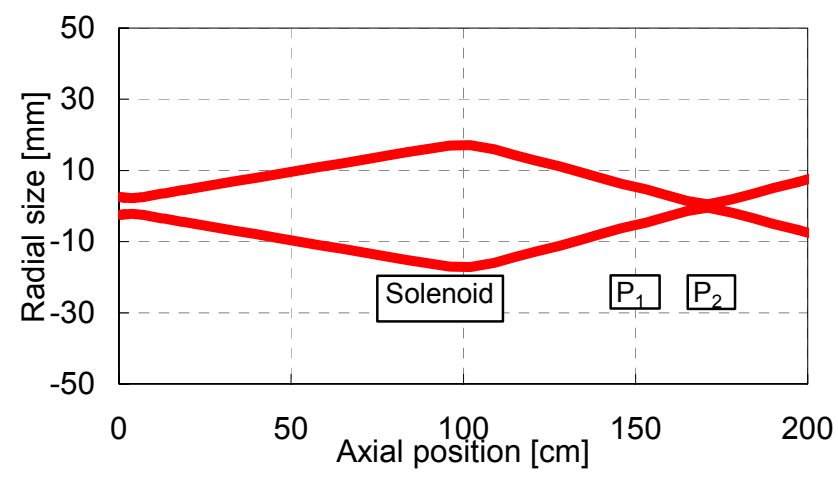

Figure 2: Beam envelope simulation using PARMELA $\mathrm{Q}=1 \mathrm{pC}, \quad \mathrm{R}_{0}, \mathrm{rms}=1.2 \mathrm{~mm}, \quad \mathrm{E}_{\max }=45 \mathrm{MV} / \mathrm{m}, \quad \mathrm{f0}=40 \mathrm{deg}$, $\mathrm{Bz}=750 \mathrm{Gs}$

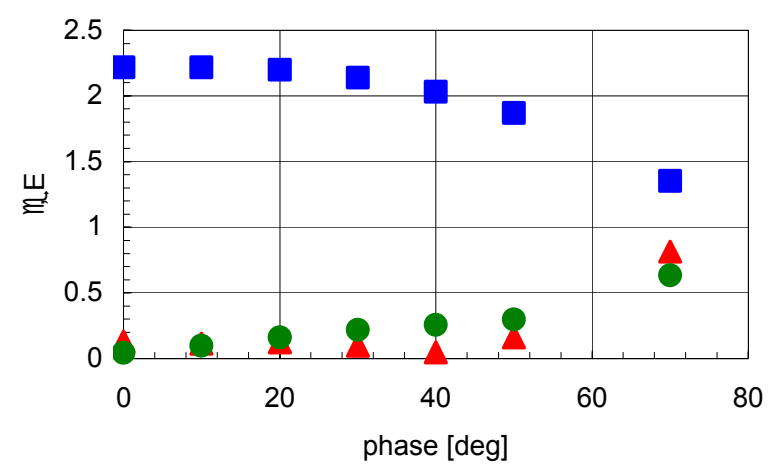

Figure 3: PARMELA simulation of longitudinal beam emittance $(\bullet)$ in $\mathrm{KeV}$ Degree, transverse emittance $(\boldsymbol{\Delta})$ in mm.mrad and energy ( $\bullet$ ) in $\mathrm{MeV}$ at different injection phase. $\quad \mathrm{Q}=1 \mathrm{pC}, \quad \mathrm{R}=1.2 \mathrm{~mm}$ (Gaussian), $\quad \mathrm{t}=6 \mathrm{ps}$, $\mathrm{E}_{\max }=45 \mathrm{MV} / \mathrm{m}$ 


\section{COMPONENTS DESCRIPTION}

The critical components of the system are the cavity, cryostat, RF system, solenoid, photocathode and laser. Specifications of each are given in the following sections RF Cavity:

The superconducting gun is a $1.3 \mathrm{GHz}$, single cell $\mathrm{Nb}$ cavity as shown in Figure 4. It is essentially one-half of an elliptical cell terminated by an end wall. The end wall has a domed shape for added stiffness, but no external stiffeners. The cavity was hydro formed from sheet RRR250 $\mathrm{Nb}$ and EB welded. The niobium cavity wall thickness is sized to $3.5 \mathrm{~mm}$ to operate with a $15 \mathrm{psi}$ pressure load on the outside of the cavity and vacuum on the inside, pass 1 watt of laser power and the RF surface thermal load through its thickness while maintaining superconducting temperatures. For this proof of principle test no cavity tuner was included. Due to the use of an end wall in the cavity, both the cavity drive port and the pickup port are on the same side of the cavity. This leads to serious cross talk during room temperature measurements, which are discussed elsewhere in these proceedings ${ }^{[2]}$. Fortunately, this problem will diminish when cool down to superconducting status.

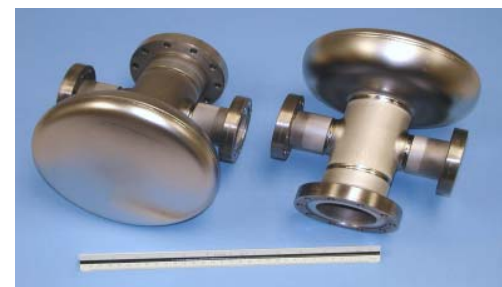

Figure 4: Photograph of $1 / 2$ cell $\mathrm{Nb}$ cavity

Earlier concepts for the cavity utilized a reentrant nose to increase the field at the cathode, thereby increasing the $\mathrm{QE}$, and the current. This proved counterproductive. The effect due to the radial $\mathrm{E}$ fields forces the beam spot size at the cathode to be reduced if the beam quality is to be maintained. The reduced beam size causes the power density from the laser to increase. Thermal simulations show that with the reentrant nose, the limit on allowable laser power density actually causes a reduction in the laser power and hence the total beam current.

\section{RF System}

The niobium cavity is expected to have an internal Q of about $7 \times 10^{9}$ in $2 \mathrm{~K}$ superconducting status. With maximum beam current the beam loaded $Q$ is $1 \times 10^{8}$. The $\mathrm{RF}$ system is designed to have an external Q around $10^{8}$. It will excite the cavity to a maximum gradient of 45 $\mathrm{MV} / \mathrm{m}$. The system consists of a $500 \mathrm{~W} \mathrm{CW}$ amplifier from PRO-COMM. The LLRF system will operate as a PLL (phase locked loop) so that its frequency will catch up with the resonant frequency of the cavity. This frequency will be divided 16 times to trigger the laser for synchronization.

\section{Laser:}

The photocathode driving laser is a commercial frequency quadrupled mode-locked $\mathrm{Nd}: \mathrm{YVO}_{4}$ laser operating at $266 \mathrm{~nm}$ with an average power of $150 \mathrm{~mW}$ and pulse duration of $\sim 6$ ps. Figure 5 shows the autocorrelator trace of the $2.3 \mathrm{eV}$ beam from this laser, while Figure 6 shows the UV beam profile. This UV beam is reflected by a vacuum metal mirror custom dielectriccoated for $266 \mathrm{~nm}$, positioned off axis in the beam line to illuminate the cavity nearly at normal incidence. The thickness of the dielectric layer is small enough to avoid charge build up yet maintain the high reflectivity. This mirror could be externally manipulated for irradiating the center of the rear surface of the cavity. Since the power density of this laser is not high enough for laser cleaning the cavity in a reasonably short time, an excimer laser will be used to irradiate the cathode surface at normal incidence for the surface preparation.

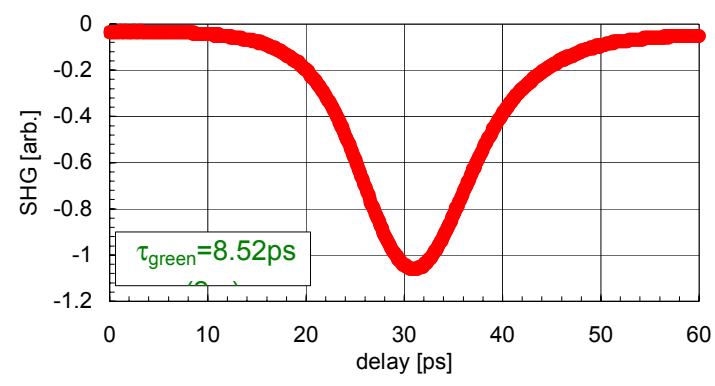

Figure 5: Autocorrelation signal of $532 \mathrm{~nm}$ radiation from the laser. In the absence of saturation, the UV pulse duration is expected to be $6 \mathrm{ps}$

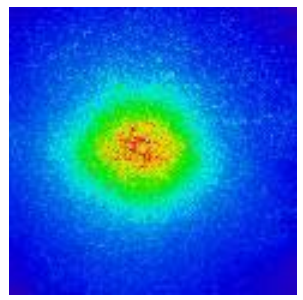

Figure 6: UV laser beam profile. 1/e diameter is $1 \mathrm{~mm}$

\section{Photocathode:}

Surface preparation of the $\mathrm{Nb}$ surface is critical to maintain high QE. Laser cleaning of either chemically etched or electro polished $\mathrm{Nb}$ can be increased by two orders of magnitude by irradiating with high intensity UV laser. Figure 7 shows the increase in the $\mathrm{QE}$ of a $\mathrm{BCP} \mathrm{Nb}$ when irradiated with a laser beam of energy density $\sim 3$ $\mathrm{mJ} / \mathrm{mm}^{2}$, pulse duration of $20 \mathrm{~ns}$ and photon energy of 5 $\mathrm{eV}$. Detailed information on laser cleaning is discussed elsewhere in these proceedings ${ }^{[3]}$. 


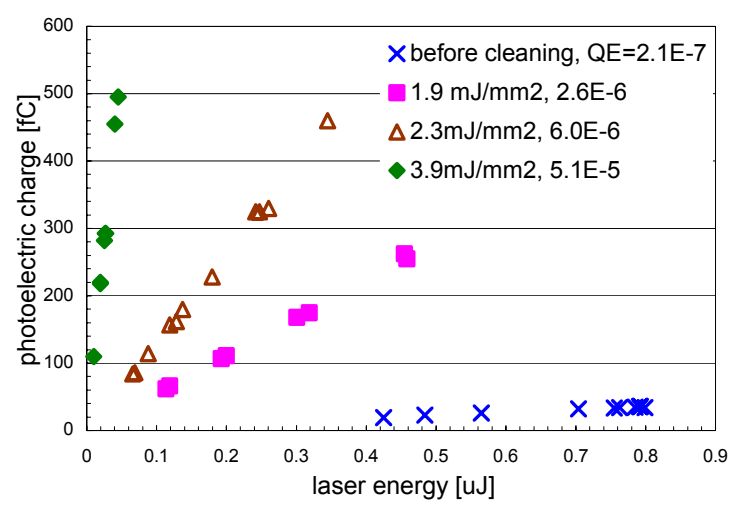

Figure 7: Electron yield of BCP sample as a function of $266 \mathrm{~nm}$ laser energy for different $248 \mathrm{~nm}$ cleaning energies. The QE of $(\star)$ is $5 * 10^{-5}$

\section{Solenoid:}

The solenoid is designed to focus the electron beam so that all the electrons can be collected in the Faraday cup or focused onto beam profile monitors placed at locations $\mathrm{P} 1$ and P2 in Figure 2 for emittance measurement. The measured field distribution along the beam axis and its dependence on the solenoid current are shown in Figures 8 and 9 respectively.

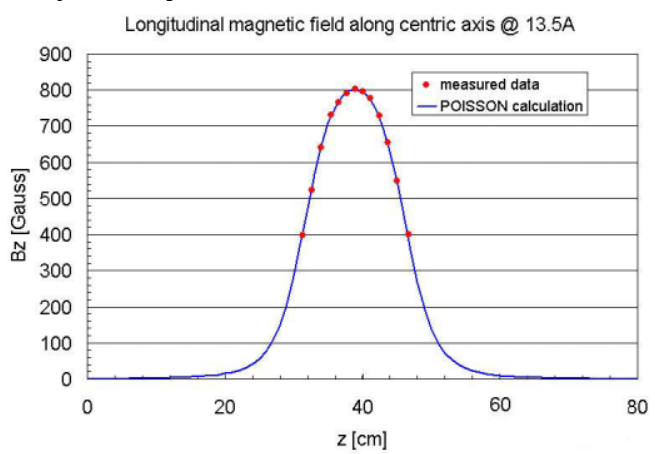

Figure 8: Longitudinal magnetic field along the center line@13.5 A

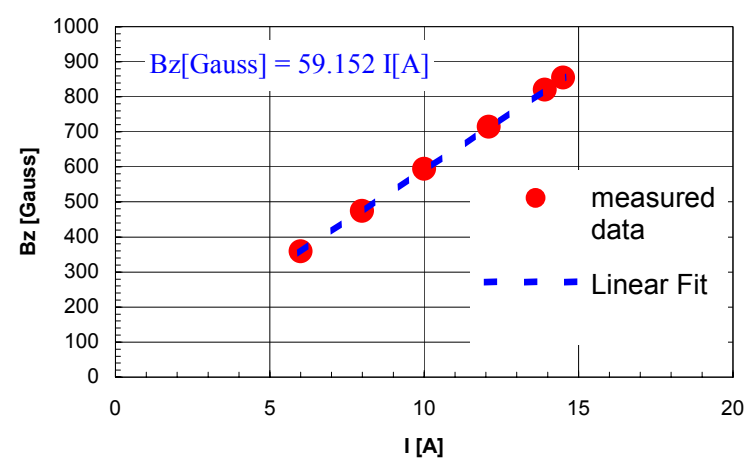

Figure 9: Measured longitudinal magnetic field as a function of solenoid current

\section{Cryostat and Cryogenic System:}

The major components of the system include the cryostat, LHe and LN2 storage dewars, mechanical vacuum pumps, cryogenic and recovery piping, vacuum piping, valving, instrumentation and controls. The cryostat consists of a stainless steel vacuum chamber, a stainless steel liquid nitrogen vessel, and a stainless steel liquid helium vessel with about 40 layers of super insulation wrapped around the nitrogen shield and $\mathrm{LHe}$ helium vessel. The cryostat is designed to hold about 60 liters of $\mathrm{LHe}$ to run experiment at $2 \mathrm{~K}$, while pumped down, for about 8 hours. The cryostat equipped with sensors to monitor inside pressure, temperatures of LHe, cavity, LN2 shield, bellow connecting inner and outer vessels. Three temperature sensors installed at the same elevation over LHe on RF cables and in He gas space. Two level probes are to measure level of $\mathrm{LHe}$ at $4.2 \mathrm{~K}$ and $2 \mathrm{~K}$ and one - level of LN2. Proportional control valve installed between cryostat and vacuum pump is designed to maintain automatically a required cavity temperature. G-10 sleeves put on RF cables are to utilize boil-off $\mathrm{He}$ flow to cool the RF cables ad exemplum gas-cooled current leads. The cryostat general arrangement and the cryostat top cover configuration are shown below in figure 10.

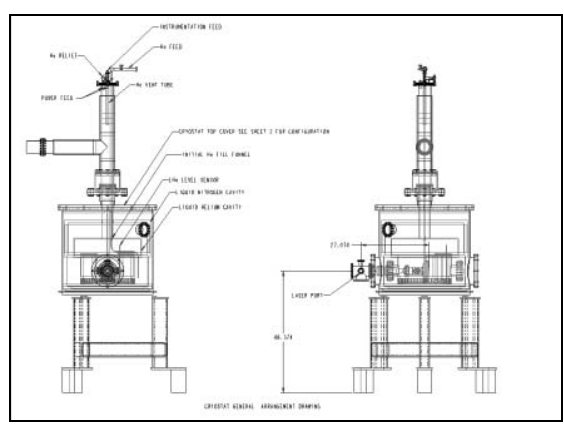

Figure 10: Section view of the cryostat

\section{Status:}

The cavity has been cooled down to $4 \mathrm{~K}$. The frequency and the $\mathrm{Q}$ of the superconducting cavity have been measured to be $1.3 \mathrm{GHz}$ and $10^{7}$. The electron beam transport has been installed. The low level RF system has been designed, built and is being tested. The laser has been characterized and the transport system is being built.

This research is supported by the U.S. Department of Energy under Contract No. DE-AC02-98CH10886, DEAC02-98CH10886DOE, and SBIR grant DE-FG0299ER82724.

\section{REFERENCES}

[1] M. Cole, et al., "Development of a Novel SC RF photocathode electron gun", LINAC'2000, p 116

[2] Y. Zhao, M. Cole, "The analysis of the cross-talk in a RF gun superconducting cavity", PAC03 paper WPAB009.

[3] Q. Zhao, T. Srinivasan-Rao, M. Cole, "Tests of Niobium Cathode for the Superconducting Radio Frequency Gun," PAC03 paper WPAB008. 